\title{
Intranasal dexamethasone: a new clinical trial for the control of inflammation and neuroinflammation in COVID-19 patients
}

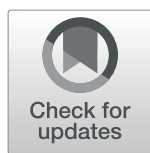

Graciela Cárdenas ${ }^{1}$, María Chávez-Canales², Ana María Espinosa ${ }^{3}$, Antonio Jordán-Ríos ${ }^{4}$, Daniel Anica Malagon ${ }^{3}$, Manlio Fabio Márquez Murillo ${ }^{4}$, Laura Victoria Torres Araujo ${ }^{4}$, Ricardo Leopoldo Barajas Campos ${ }^{4}$, Rosa María Wong-Chew ${ }^{5}$, Luis Esteban Ramirez González ${ }^{6}$, Karent lbet Cresencio ${ }^{6}$, Enrique García Velázquez ${ }^{6}$, Mariana Rodriguez de la Cerda ${ }^{6}$, Yoana Leyva ${ }^{4}$, Joselin Hernández-Ruiz ${ }^{3}$, María Luisa Hernández-Medel ${ }^{3}$,

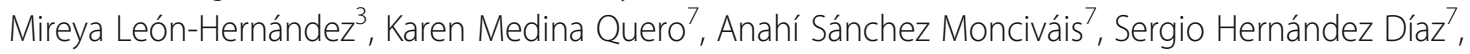
Ignacia Rosalia Zeron Martínez ${ }^{7}$, Adriana Martínez-Cuazitl $\left.\right|^{7}$, Iván Noé Martínez Salazar ${ }^{7}$, Eduardo Beltrán Sarmiento ${ }^{7}$, Aldo Figueroa Peña ${ }^{7}$, Patricia Saraí Hernández ${ }^{7}$, Rafel Ignacio Aguilar Reynoso ${ }^{7}$, Daniela Murillo Reyes ${ }^{7}$, Luis Rodrigo del Río Ambriz ${ }^{7}$, Rogelio Antonio Alfaro Bonilla7, Jocelyn Cruz ${ }^{7}$, Leonor Huerta ${ }^{8}$, Nora Alma Fierro ${ }^{8}$, Marisela Hernández ${ }^{8}$, Mayra Pérez-Tapia ${ }^{9}$, Gabriela Meneses $^{10}$, Erick Espíndola-Arriaga ${ }^{8}$, Gabriela Rosas $^{11}$, Alberto Chinney ${ }^{6}$, Sergio Rosales Mendoza ${ }^{12}$, Juan Alberto Hernández-Aceves ${ }^{8}$, Jaquelynne Cervantes-Torres ${ }^{8}$, Anai Fuentes Rodríguez ${ }^{13}$, Roxana Olguin Alor ${ }^{13}$, Sandra Ortega Francisco ${ }^{13}$, Evelyn Alvarez Salazar ${ }^{13}$, Hugo Besedovsky ${ }^{14}$, Marta C. Romano ${ }^{15}$, Raúl J. Bobes ${ }^{8}$, Helgi Jung ${ }^{16}$, Gloria Soldevila ${ }^{13}$, Juan López-Alvarenga ${ }^{17}$, Gladis Fragoso ${ }^{8}$, Juan Pedro Laclette ${ }^{8}$ and Edda Sciutto ${ }^{8^{*}}$ (D)

\footnotetext{
Abstract

By end December of 2021, COVID-19 has infected around 276 million individuals and caused over 5 million deaths worldwide. Infection results in dysregulated systemic inflammation, multi-organ dysfunction, and critical illness. Cells of the central nervous system are also affected, triggering an uncontrolled neuroinflammatory response. Low doses of glucocorticoids, administered orally or intravenously, reduce mortality among moderate and severe COVID-19 patients. However, low doses administered by these routes do not reach therapeutic levels in the CNS. In contrast, intranasally administered dexamethasone can result in therapeutic doses in the CNS even at low doses.
}

\footnotetext{
* Correspondence: edda@unam.mx

${ }^{8}$ Departamento de Inmunología, Instituto de Investigaciones Biomédicas, Universidad Nacional Autónoma de México, Circuito escolar s/n, 04510 Mexico, Mexico

Full list of author information is available at the end of the article
}

(c) The Author(s). 2022 Open Access This article is licensed under a Creative Commons Attribution 4.0 International License, which permits use, sharing, adaptation, distribution and reproduction in any medium or format, as long as you give appropriate credit to the original author(s) and the source, provide a link to the Creative Commons licence, and indicate if changes were made. The images or other third party material in this article are included in the article's Creative Commons licence, unless indicated otherwise in a credit line to the material. If material is not included in the article's Creative Commons licence and your intended use is not permitted by statutory regulation or exceeds the permitted use, you will need to obtain permission directly from the copyright holder. To view a copy of this licence, visit http://creativecommons.org/licenses/by/4.0/ The Creative Commons Public Domain Dedication waiver (http://creativecommons.org/publicdomain/zero/1.0/) applies to the data made available in this article, unless otherwise stated in a credit line to the data. 
Methods: This is an approved open-label, multicenter, randomized controlled trial to compare the effectiveness of intranasal versus intravenous dexamethasone administered in low doses to moderate and severe COVID-19 adult patients. The protocol is conducted in five health institutions in Mexico City. A total of 120 patients will be randomized into two groups (intravenous vs. intranasal) at a 1:1 ratio. Both groups will be treated with the corresponding dexamethasone scheme for 10 days. The primary outcome of the study will be clinical improvement, defined as a statistically significant reduction in the NEWS-2 score of patients with intranasal versus intravenous dexamethasone administration. The secondary outcome will be the reduction in mortality during hospitalization.

Conclusions: This protocol is currently in progress to improve the efficacy of the standard therapeutic dexamethasone regimen for moderate and severe COVID-19 patients.

Trial registration: ClinicalTrials.gov NCT04513184. Registered November 12, 2020. Approved by La Comisión Federal para la Protección contra Riesgos Sanitarios (COFEPRIS) with identification number DI/20/407/04/36. People are currently being recruited.

Keywords: Dexamethasone, Intranasal administration, Inflammation, Neuroinflammation, COVID-19

\section{Graphical abstract}

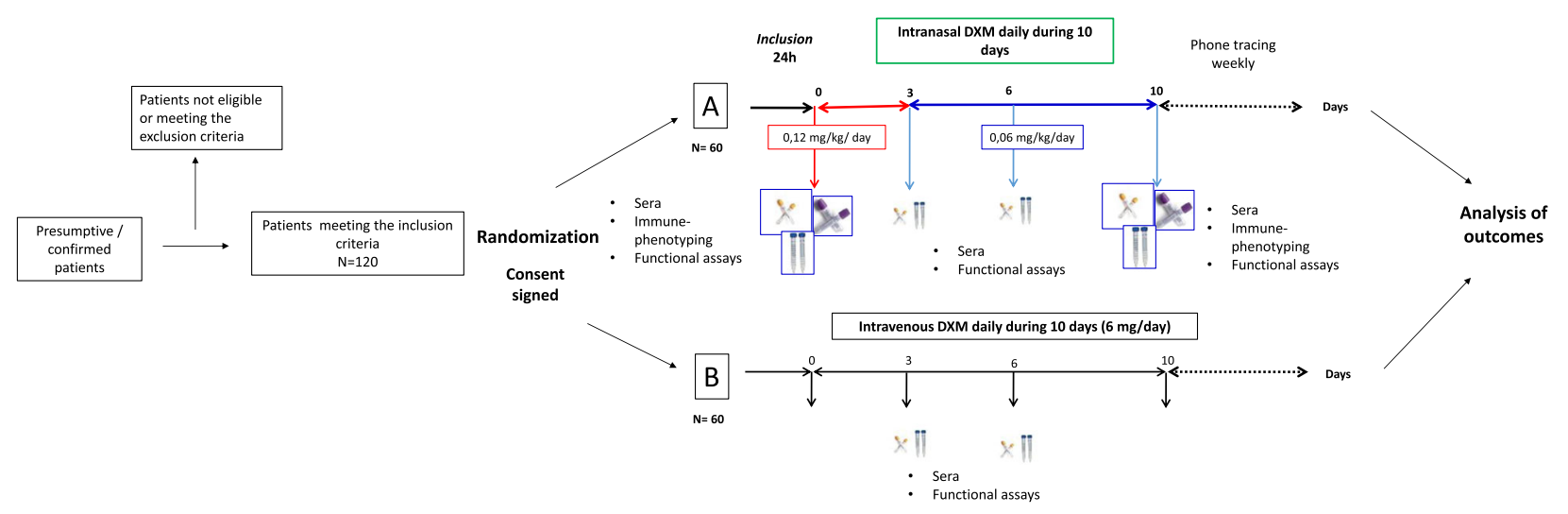

\section{Highlights}

- REVIVAL is a multicenter, open-label, randomized, controlled study to compare the standard low doses of intravenous dexamethasone with weight-adjusted low doses of intranasal dexamethasone.

- Intranasal dexamethasone can reach the respiratory tract more effectively than intravenous administration.

- Intranasal dexamethasone can reach the central nervous system in therapeutic concentrations, even at low doses.

- REVIVAL aims to reduce central failures and sequelae by controlling not only systemic inflammation but also neuroinflammation.

Keywords: Dexamethasone, Intranasal administration, Inflammation, Neuroinflammation, COVID-19

\section{Background}

So far, the outbreak of COVID-19 has infected around 276 million individuals and caused over 5 million deaths worldwide (https://coronavirus.jhu.edu/map.html), with a current global case-fatality ratio of $2 \%$. The most affected geographic region is the Americas, with a casefatality ratio of $2.4 \%$.

Several factors predict a poor outcome for COVID-19 patients. These include comorbidities (diabetes, hypertension, obesity) and aging, which are normally accompanied by a dysregulated inflammatory response [1]. Other relevant factors include SARS-CoV-2 neurotropism/neuroinvasive [2-9] as viral RNA was found in the brain of patients who deceased from severe acute respiratory syndrome due to COVID-19 infection [10-12]. Likewise, evidence of astrocytic activation and neuronal damage was reported in severe COVID-19 patients with elevated plasmatic levels of glial fibrillary acidic protein and neurofilament light polypeptide 
[13]. Other authors have shown extensive infection of astrocytes [14] and neurons in $2 \mathrm{D}$ and $3 \mathrm{D}$ cultures $[15,16]$. The infection of cells of the central nervous system (CNS) results in the expression of pathogen-associated molecular patterns (PAMPs) and danger-associated molecular patterns (DAMPs) that trigger a neuroinflammatory response. The exacerbated systemic inflammation, combined with the consequent breakdown of the blood-brain barrier, induces the migration of cells and peripheral inflammatory mediators into the brain. Together, these factors intensify and sustain neuroinflammation, which, added to peripheral damage, may contribute to multi-organ dysfunction and death [10, 12].

\section{Natural history of SARS-CoV-2 infection}

A clinical staging system for SARS-CoV-2 infection has been proposed. It involves four stages: early infection (Stage I, mild), pulmonary involvement without hypoxia (Stage IIa, moderate), or with hypoxia (Stage IIb), and systemic hyperinflammation (Stage III) [17] (Fig. 1).

After exposure to SARS-CoV-2, the virus enters the host through the nasal cavity and respiratory airway. Early infection (Stage I) courses with mild and nonspecific symptoms (fever, malaise, and asthenia); upon this prodromic phase, the virus binds to its target receptors ACE2, TMPRSS2 [18, 19], and NRP-1, the most recently discovered target $[20,21]$. These receptors are highly present in several tissues, including the olfactory neuroepithelium, although to a lesser extent in the sensory olfactory neurons, and the lung [19-22]. Thus, the infection can be established in the lungs (Stage II), leading to viral pneumonia, cough, and fever with or without hypoxia. In the lung, SARS-CoV-2 PAMPs will be recognized by endosomal TLR3, TLR7, and TLR8, and cytosolic RIG-I-like receptors [23]. The virus can also reach the CNS through the olfactory and trigeminal nerve terminals. Once in the CNS, it can infect and damage the endothelium, pericytes, and neural cells that express ACE2 and NRP-1 receptors [20, 21], promoting neuroinflammation (Fig. 1). CNS viral involvement is related to headache, dizziness, and ataxia, but infection also may progress to the whole brain, including the brainstem [5, 6]. In a minority of infected patients, the disease progresses to Stage III, coursing with a hyperinflammatory syndrome. This syndrome is characterized by the sustained production of pro-inflammatory cytokines (including IL-1 $\beta$ and TNF $\alpha$ ) and reactive oxygen species

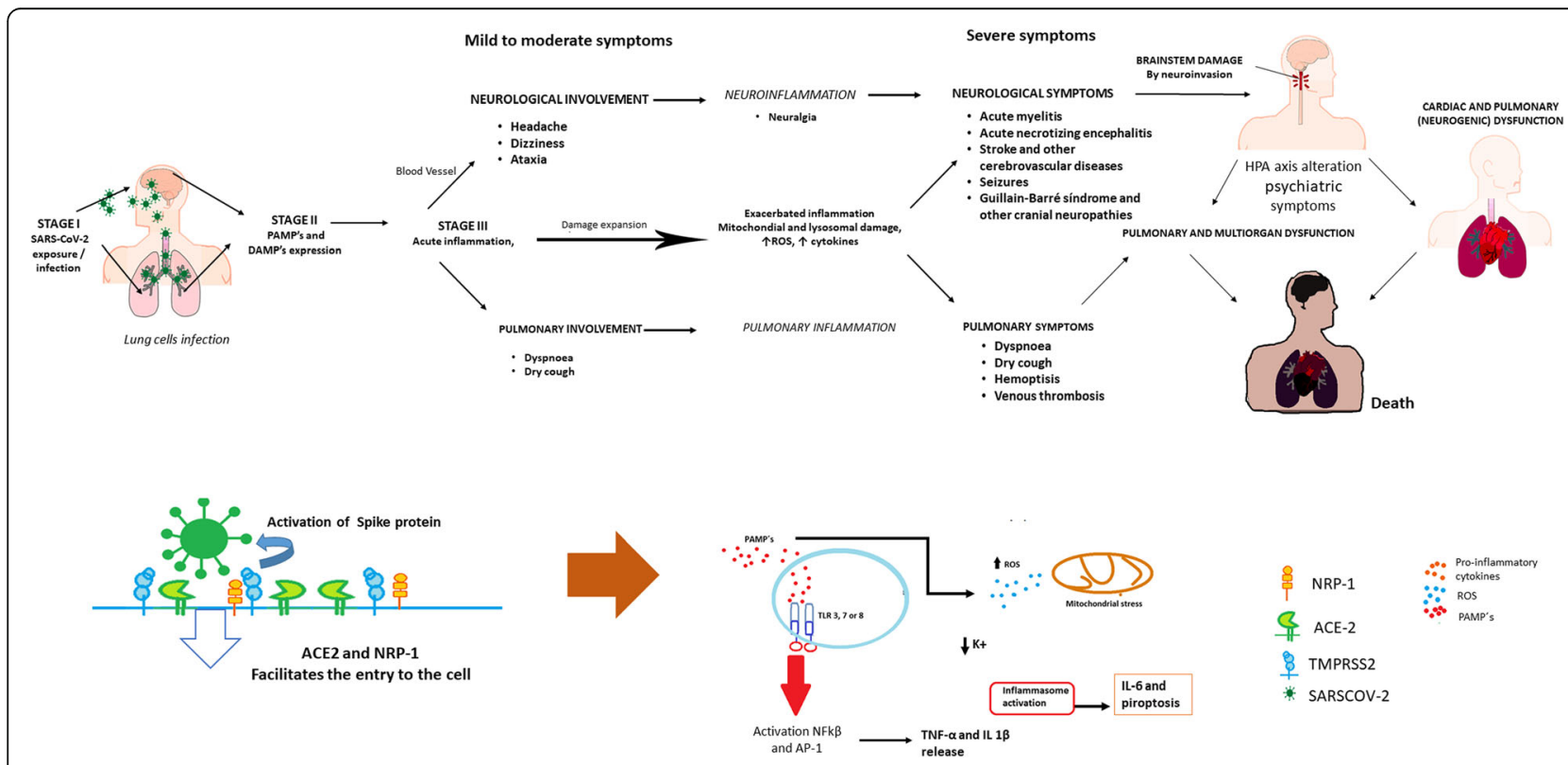

Fig. 1 Inflammatory phenomena associated with SARS-CoV-2 infection and its neurological and respiratory manifestations. The SARS-CoV-2 virus mainly enters the respiratory tract and reaches the lungs through direct ventilation and the CNS through the olfactory and trigeminal nerves. The entry of the virus is facilitated by NRP-1, ACE2 receptors, and protein S activation by TMPRSS2. In the CNS, the virus infects neurons, glial cells, and endothelial cells, increasing the permeability of the BBB. This may cause cerebral edema, intracranial hypertension, and neuroinflammation. If the viral infection continues, the damage spreads throughout the body, causing heart and systemic failure. This damage is associated with increased neuroinflammation directed by microglia and oligodendrocytes, causing damage to the brain stem and dysfunction of the heart and lungs. The exacerbated inflammation and intravascular coagulation induce respiratory arrest, possibly leading to the patient's death. The inflammation is triggered by viral components (PAMPS) that activate TLR3, 7, and 8 receptors on the cell surface. Consequently, there is an increased production of pro-inflammatory cytokines (TNFa and IL 1 $\beta$ ) and ROS, which can modify the P2X7 receptor in the brain and activate the inflammasome by the decrease of $\mathrm{K}^{+}$. The activation of the inflammasome increases the production of IL- 6 and pyroptosis. This diagram is based on the knowledge at the time of writing the manuscript 
(ROS), mitochondrial and lysosomal damage, and the hyperactivation of P2X7 receptors. These processes induce the activation of the inflammasome, which increases IL-6 and leads to pyroptosis. Consequently, the dissemination of viral antigens and RNA into the circulation establishes a persistent inflammatory cycle. Immune complexes can also be generated and deposited in target organs [23-25]. During this phase, sustained neuroinflammation may exacerbate the neuronal injury, spreading damage and contributing to central respiratory failure, ultimately resulting in multi-organ dysfunction [17].

A crucial strategy to treat COVID-19 patients is controlling neural and systemic inflammation. For this purpose, it is essential to consider how viruses invade the human organism. The intranasal route is the most frequent; it allows direct access to both respiratory and central nervous systems through neural pathways [5, 15-18]. Coronaviruses, including SARS-CoV-2, can infect brainstem neurons associated with cardio-respiratory control; thus, pulmonary function is also altered at the central level [5, 26-29]. COVID-19 neurological clinical symptoms, particularly nausea, vomiting, and dysgeusia, seem to involve the dorsal vagal complex (DVC) and the nucleus tractus solitarius (NTS), linked to the control of several autonomic functions [26]. The NTS is also a well-known target of neuro-immune activation [30]. Its ascending projections reach the hypothalamic paraventricular nucleus, involved in the activation of the HPA axis, and the rostral ventrolateral medulla (RVM), which controls respiratory and cardiovascular functions [31].

The viral infection in the respiratory and central nervous systems promotes the expression of PAMPs and DAMPs. These signals trigger the inflammasome and oxidative stress $[23,32]$. Later during infection, the inflammatory response may become dysregulated, extending the initial damage caused by the infection.

\section{Adrenal insufficiency in SARS-CoV-2 infection}

Critically ill patients affected by different pathologies frequently show adrenal insufficiency, which may increase morbidity and mortality [33, 34]. COVID-19 might affect the hypothalamic-pituitary-adrenal (HPA) axis as well. Hypothalamic and hypophyseal tissues express ACE2 and can therefore be viral targets [35]. The virus may directly damage the hypothalamus and the pituitary, leading to hypothalamic-pituitary dysfunctions.

Since the SARS outbreak of 2003, autopsy studies have demonstrated that coronaviruses affect the HPA axis and promote vasculitis in several organs, including adrenal glands; in particular, adrenal cortical cells undergo degeneration and necrosis [36]. Although the full longterm spectrum of COVID-19 endocrine manifestations is still unclear, several endocrine alterations have been reported in SARS survivors. These include hypocortisolism, hypothyroidism, and low levels of dehydroepiandrosterone, suggesting a transient hypothalamicpituitary dysfunction [37]. Recently, an Arabian study including 28 patients reported the adrenal response to an acute COVID-19 infection; the median level of morning cortisol was $196(31-587) \mathrm{nmol} / \mathrm{L}$, and the median level of ACTH was 18.5 (4-38 ng/L). Interestingly, patients with severe disease had lower cortisol and ACTH [38]. In addition, other autopsy studies have found edema, neuronal degeneration, and evidence of viral genome in the hypothalamus [39]. Thus, in the presence of subacute thyroiditis or adrenal insufficiency, corticosteroid therapy should help by reducing high amounts of thyroid hormones and replace adrenal function, improving the evolution of these patients regardless of the route of administration.

\section{Rationale}

Dexamethasone sodium phosphate (ALIN, injectable solution. Chinoin Laboratory) is a highly soluble glucocorticoid with a $\mathrm{pH}=7-8.5$ that does not harm the nasal mucosa. This synthetic steroid is an antiinflammatory and immunomodulatory drug that inhibits platelet activation, prostaglandin and leukotriene synthesis, and coagulation by regulating transcriptional factors like NF- $\kappa B$ and AP-1 [40-42]. Furthermore, DXM exerts important neuroprotective effects such as rescuing neurovascular integrity during neuroinflammation [43].

\section{Dexamethasone: a potent anti-inflammatory drug}

Considering that the complications of COVID-19 result from exacerbated peripheral and neural inflammation derived from the so-called cytokine storms, at least three key points have been addressed in the use of DXM for the treatment of Coronavirus patients: timing, dose, and route of administration. First, the drug should not be applied from the beginning of the infection when inflammation favors the control of viral replication and the establishment of an adaptive immune response that serves to control the infection. A low dose of DXM (6 $\mathrm{mg}$ per patient for 10 days) has quickly and effectively controlled pulmonary inflammation with minimal negative side effects [44]. In addition, the intranasal route would allow direct access of DXM to the CNS through the olfactory and trigeminus nerves, thereby controlling the sustained neuroinflammation provoked by damage to infected astrocytes, neurons, and microglia. Therefore, cardiac and central respiratory failure could be diminished in COVID-19 patients, avoiding fatalities.

In experimental models, it is well known that drugs administered intranasally usually grant higher bioavailability in the CNS compared with similar doses administered intravenously, since intranasal drugs bypass the 
BBB and hepatic degradation [45-48]. In addition, intranasal DXM might control inflammation by arriving directly to the respiratory system more effectively and quickly than the intravenous route [46-49]. DXM prevents the binding of ACE2 to the spike protein of SARSCoV-2 and can also bind to LYS353, an active residue of the receptor-binding domain (RBD) [50]. Moreover, DMX reduces ACE2 expression in several cell types by suppressing type I interferon expression [51]; it can also downregulate neutrophil extracellular traps (NETs), possibly through regulating Toll-like receptors [52]. Hyperinflammation is related to high levels of NETs and neutrophilia, which, in turn, predicts thrombosis and poorer outcomes in acute respiratory distress syndrome (ARDS) [53, 54].

\section{Methods}

\section{Trial design}

The "REVIVAL" trial is an interventional, phase 2, multicenter, open-label, randomized controlled study in adult patients with confirmed COVID-19 diagnosis, designed to evaluate the efficacy (superiority) of low doses of intranasal DXM compared to intravenous administration (allocation ratio 1:1) in patients of five COVID-19 referral centers in Mexico City.

\section{Settings and trial sponsor}

This clinical trial is being conducted at the five Health Institutions in Mexico City: "Hospital General de México Dr. Eduardo Liceaga," "Instituto Nacional de Neurología y Neurocirugía Manuel Velasco Suárez," "Instituto Nacional de Cardiología Ignacio Chavez," "COVID-19 unit at Citibanamex," and "Hospital Central Militar."

The Hospital General de México Dr. Eduardo Liceaga (HGMEL) is the trial sponsor investigator which is in charge of initiating, administering, and monitoring the current clinical trial. The hospital address is Dr. Balmis 148, Doctores, Cuauhtemoc, CP06720, Mexico City and the telephone is $(+52) 5527892000$. The current protocol is already approved by HGMEL (\#DI/20/407/043).

When recruitment of all participants be completed, the trial sponsor investigator will also participate in the interpretation of data, writing the paper, and the decision to submit the report for publication.

\section{Eligibility criteria}

Inclusion criteria comprise patients of both sexes (nonpregnant female), aged from 18 to 90 years old, with presumptive SARS-CoV-2 infection and more than 5 days of clinical evolution, with moderate to severe symptoms requiring oxygen support or high flux mechanical ventilation (NEWS-2 $\geq 5$ ), and abnormal CT-chest scan CORADS $>3$. Patients should be diagnosed with atypical pneumonia, confirmed by chest images and oxygen saturation $\left(\mathrm{SpO}_{2}\right)$ lower than $93 \%$ in ambient air or a ratio of the partial pressure of oxygen and the fraction of inspired oxygen $\left(\mathrm{PaO}_{2}: \mathrm{FiO}_{2}\right)$ equal to or lower than 300 $\mathrm{mmHg}$, and a positive RT- PCR SARS-CoV-2 test. These patients will be allocated into the experimental group or the control group in a 1:1 ratio (two arms) (Fig. 2) according to the randomization.

Exclusion criteria include patients with an RT-PCR SARS-CoV-2 negative test; previously receiving GCs at high doses by oral or intravenous administration; severely immunosuppressed as in AIDS, pregnancy, and autoimmune diseases; those who have received outpatient treatment with steroids for more than $72 \mathrm{~h}$ before hospital admission; those older than 90 years; with DXM allergy; risk for glaucoma; or recurrent respiratory diseases. Patients receiving other monoclonal antibodybased treatments such as tocilizumab are also excluded. No other concomitant treatments are prohibited for patient eligibility.

Elimination criteria include voluntary withdrawing, lack of informed consent letter, or imminent risk of death within $48 \mathrm{~h}$.

\section{Interventions \\ Groups and comparators}

The study will be carried out in two groups; group A (experimental) will receive intranasal DXM, and group B (Control) will receive intravenous DXM (Fig. 2). This experimental design is based on previously reported data indicating that intranasal administration can reach the brain and bloodstream quickly and efficiently [46-49]. Group A will receive intranasal DXM daily, at the dose of $0.12 \mathrm{mg} / \mathrm{kg}$ for the first 3 days, followed by $0.06 \mathrm{mg} / \mathrm{kg}$ for 7 days. Group B will receive $6 \mathrm{mg}$ of intravenous DXM daily. In each hospital, the Pharmacovigilance staff will collect the adverse event information, for any (untoward o abnormal) medical manifestation, symptom, or disease, whether or not related with the drug treatment in each patient throughout the study.

\section{Procedures}

After randomization, a Case Report Format (CRF) (printed or electronic) for each patient will be filled daily by the medical staff and completed at the end of treatment or fatal outcome, whatever occurs first. Blood and saliva samples will be collected every third day during the whole treatment to perform ancillary tests such as SARS-CoV-2 viral load, functional immunological assessment (lymphocyte cytometry, cytokine/chemokine profile), and cortisol levels, among other analyses.

All biological samples will be each $48 \mathrm{~h}$ at every hospital center participant, transported by a specialized service and concentrated at Instituto de Investigaciones Biomédicas (UNAM) to be managed and stored at - 


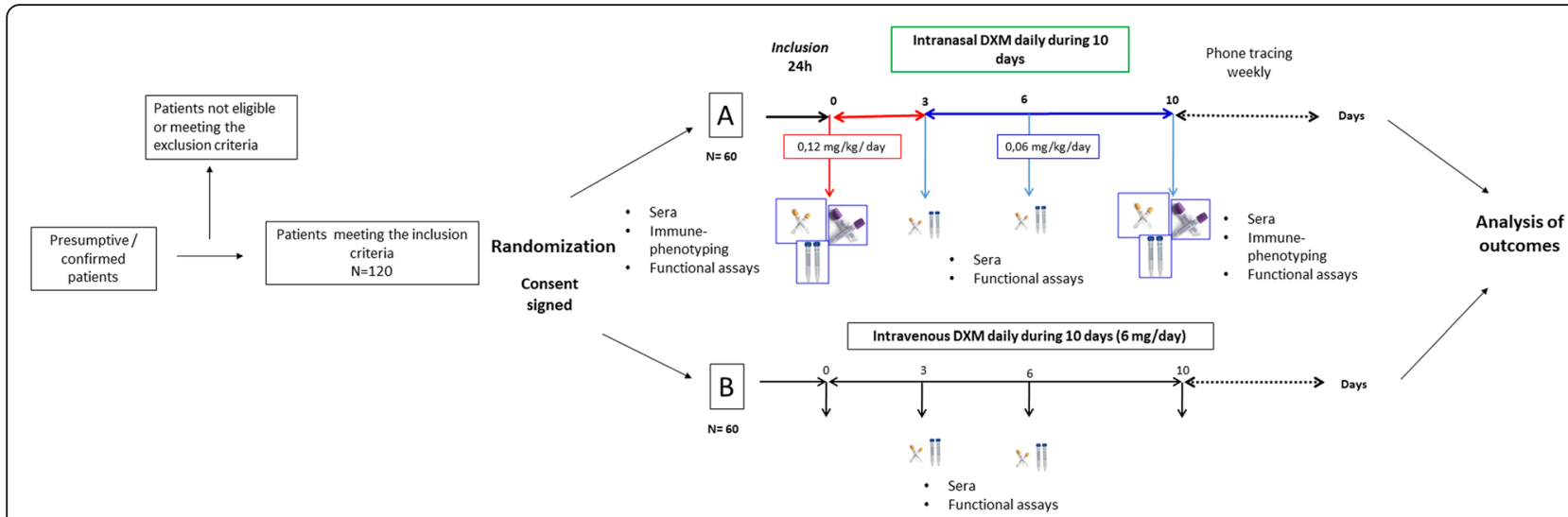

Fig. 2 Outline of the REVIVAL trial clinical protocol. Initially, patients will be informed about the clinical trial; if they accept and sign the consent, they will be randomized using the Sealed envelope ${ }^{\circledR}$ software. Group A will receive intranasal DXM; Group B will receive intravenous DXM. Both groups will be sampled on days $0,3,6$, and 10 post-treatment to collect sera and nasopharyngeal swabs. Patients will be monitored throughout the study. The results will be tested for statistical differences between groups

$70^{\circ} \mathrm{C}$ until use. As mentioned in the Informed Consent, all samples will be used for the next 3 years. Additional biological samples may be taken at each hospital center when necessary, according to the treating physicians and will not be part of this protocol.

All personal data and medical information of the patients will be treated in a strictly confidential way. Only the lead investigator and the hospital coordinator investigators will have access to this information.

\section{Participants}

The sample includes 120 adult patients of both sexes between 18 and 90 years of age, with moderate and severe forms of COVID-19. Participants are recruited from the five participant hospitals. Medical teams at each participating Hospital Center will be led by a responsible clinician who will obtain the informed consent letter. All eligible patients or a responsible family member (in the case that the patient cannot sign) receives an informed consent letter from the lead clinician, where the characteristics of the procedure are detailed. A medical team at each hospital will oversee the enrollment of participants according to a randomization scheme. A clinical monitor will supervise the data generation, protocol implementation, and appropriate patient enrollment.

\section{Sample size and randomization}

The analysts of the statistic team calculated the size with EPIDAT version 3.1.2 software, using the option "Sample size and surveillance curves" with an estimated 50\% increase in the proportion of patients free of mechanical ventilation [intranasal DXM $70 \%$ vs. intravenous DXM 45\%]. This value was estimated using the data of the COVID-19 patients registered in Mexican hospitals with a confidence of $95 \%$, power of $80 \%$, and proportion of losses of $10 \%$. A sample size of 60 patients per group was obtained with these parameters. The randomization will be made with Sealed Envelope software. This software is freely available from https://www.sealedenvelope. com/simple-randomiser/v1/lists [Accessed May 5, 2020]. This study is a multicenter randomized controlled trial (Fig. 2).

\section{Confidentiality}

Each patient who agrees to participate in the protocol will be assigned an identification number that will be used throughout the procedure. This code distinguishes the hospital of origin and the patient's identification number. All the information collected during the procedure will be confidential (following the data privacy statement found in the informed consent letter) and used only for research purposes and follow-up of adverse effects.

\section{Outcomes}

The expected primary outcome is clinical improvement, defined as a two-point improvement in the ordinal scale regarding the initial NEWS-2 score. This is an adapted score recommended by the World Health Organization (WHO) and the National Institute for Health and Care Excellence (NICE) to facilitate the early recognition and escalation of deteriorating patients $[55,56]$. Although this score is commonly adopted for triage, it can also be used for continuous assessment, particularly in predicting intrahospital mortality. In this context and based on previous experimental data regarding the sustained effect of intranasal dexamethasone, we decided to evaluate the improvement in the initial NEWS-2 score as the first outcome.

The secondary expected outcome includes a reduction in mortality that will be examined during treatment (after randomization), a reduction of the time required 
for mechanical ventilation, the length of patient's stay in the hospital, 30-day post-discharge mortality, and intrahospital complications. Viral load, the immuneinflammatory profile, and other physiological parameters will also be evaluated before and after treatment (see above).

\section{Data collection and management}

After acceptance and signature of the informed consent letter, patients will be randomized, saliva and nasopharyngeal samples will be taken to measure the viral load, and treatment will begin as indicated in Fig. 2. The patient's clinical history will be based on the initial results and physical inspection. Blood and saliva samples will be collected before the start of DXM treatment and at days 3,6 , and 10 within the treatment period. All statistical analyses will be performed by our team of specialists.

The samples will be sent for specialized analysis following standardized operating procedures (SOP's). All patient data, including clinical history and laboratory data, will be collected daily and managed using REDCap (Research Electronic Data Capture System) tools hosted at Instituto de Investigaciones Biomédicas, UNAM [57, 58].

\section{Plans to promote participant retention and complete follow-up}

All participants will receive specialized medical care, including clinical, neurological, and neuropsychological studies. These evaluations will be carried out 1, 3, 6, and 12 months after COVID-19 to monitor the evolution of the disease. The participants that present any functional post-COVID decline will receive medical treatment and neurorehabilitation.

Likewise, patients who present an adverse effect or health problem during their participation in this study or derivate to it upon hospitalization will receive all necessary treatment and care until their resolution in the "Hospital General Hospital de Mexico Dr. Eduardo Liceaga." In addition, patients will be monitored every 3 months for 1 year after the study. During the study, other immunomodulatory drugs such as colchicine, GM-CSF inhibitors, IVIG, interferons, interleukins inhibitors, and kinase inhibitors can be used under the responsibility of the physician.

\section{Data monitoring committee}

A multidisciplinary group of independent experts will be on charge to assess the progress, safety data, and if needed, critical efficacy endpoints of this study.

\section{Data management}

The information collected during the procedure will be documented physically and digitally in an exact and precise manner. Researchers will use complete patient reports along with the molecular and immunological tests to analyze the outcomes. The information collected will be treated as confidential, and only the global results will be published without showing the patients' names. In case data are required, the information can be requested from the researchers with valid reasons.

\section{Statistical analysis}

Descriptive statistics using mean (standard deviation) or frequency (percentage) will be used following the dimension scale of the variable. An initial comparison between the hospital will define relevant differences among them. Since healthcare conditions are variable in each hospital included in this study, we will analyze the results with a multilevel mixed longitudinal model. The outcome analysis for NEWS-2 components (i.e., respiration rate, oxygen saturation, systolic blood pressure, pulse rate) will be done using the last observation carried forward (LOCF) in those subjects with at least two observations, this analysis will avoid over-optimistic estimates of efficacy. Other efficacy variables like biochemistry or cell counts will be analyzed without ITT due to the susceptibility to type II error.

Biochemical variables and cell counts will be aggregated using principal component analysis and use the scores if the Kaiser-Meyer-Olkin (KMO) is greater than 0.6 and sphericity $p<0.0001$. The scores using varimax rotation will be used as dependent variables if the method is suitable for our data. The longitudinal mixed model can be described as:

$$
y_{i j}=X_{i j} \beta+u_{i}+v_{j(i)}+\epsilon_{i j}
$$

where $j=1, . ., \mathrm{Ti}$ are nested in 10 time points, within $i$ $=1, . ., 5$ hospitals. The Xs correspond to fixed variables like the treatments, age, sex, and weight, among other confounders.

A database in vertical format was reshaped to nest the biochemical, inflammatory markers, cell count adjusted by sex, age, and BMI. We will perform restricted maximum likelihood (REML) estimation in case we obtain balanced samples. However, if we obtain an unbalanced sample, we will use maximum likelihood instead [59]. Because we expect a small sample size from some hospitals, we will make inferences using the Kendward-Roger degrees of freedom method. We will make simultaneous inferences about 1 linear combinations of fixed factors. Despite the complicated situation where $l>1$ we will consider:

$$
F=\frac{1}{l}(\hat{\beta}-\beta)^{T} L\left(L^{T} \hat{\phi}_{A} L\right)^{-1} L^{T}(\hat{\beta}-\beta)
$$

The internal random structure $\left[L^{T} \hat{\phi}_{A} L\right]$ can change in different settings, but still will be a good approximation. 
Simulations studies showed adequate performance of modifications with different REML [60]. Finally, the structure of residual error will be analyzed within the lowest-level group. Once recognized, the covariance structure we will use unstructured, Toeplitz, or exponential corrections. This approach will give unbiased approximation even in presence of unbalanced samples.

The residuals for our mixed models will be considered by the source of present variability: The marginal residuals will predict the marginal errors, the conditional residuals, and the best linear unbiased predictor (BLUP) residuals. The conditional residuals will be standardized with:

$\hat{\varepsilon}_{k}^{*}=\frac{\hat{\varepsilon}_{k}}{\sigma \sqrt{p_{k k}}}$, where $p_{k k}$ represents the $k$ th element of the covariance structure. These residuals are functions of the joint leverage of fixed and random effects.

Subgroup analysis will be based on interaction as secondary analysis. This information will help to identify multiplicative effects from basal conditions like the presence of type 2 diabetes, obesity, hypertension, clinical history IECA medication, or other conditions.

All analysis will describe the number of patients with the outcome of interests, the treatment effect (mainly using eta squared), additive and multiplicative interactions with 95\% confidence intervals, and the direction of the interaction (positive or negative) by specific subgroups. The analysis will be performed with Stata version 17.0 [StataCorp LLC College Station TX]. A statistical difference with $P<0.05$ will be considered significant.

\section{Interim analysis}

This analysis will be performed by an independent expert team to examine some relevant data as baseline, safety outcome, and efficacy outcome data to consider ending the study if no trend is observed

\section{Conclusions}

Intranasal DXM at low doses could be a more effective therapeutic option to control peripheral and central inflammation during ARDS in severe and critical forms of SARS-CoV-2 infection. In addition, it could stabilize the activity of the HPA axis upon this severe stress condition. Although low-dose systemically administered DXM is beneficial for COVID-19 patients, it cannot reach effective therapeutic concentrations in the CNS to control neuroinflammation. In contrast, intranasal administration of DXM is highly effective in controlling neuroinflammation, as demonstrated in experimental inflammation models [41-44]. Therefore, in the REVIVAL trial clinical protocol, we propose boosting the effect of low-dose DXM treatment through an intranasal route of administration. This route will allow reaching the CNS at therapeutic doses that may effectively reduce the morbidity and mortality in severe or critical COVID19 patients, further than that reported in the RECOVERY trial.

Low doses of intranasal DXM are currently being tested (clinicaltrials.gov id: NCT04513184) in a randomized study in hospitalized COVID-19 patients (moderate and severe forms). The clinical evolution and respiratory parameters of the patients receiving intranasal DXM (experimental treatment) are compared with those of patients receiving $6 \mathrm{mg}$ of intravenous DXM, the currently recommended treatment (https://www.covid1 9treatmentguidelines.nih.gov/). Considering the prevalence of metabolic syndrome and obesity in Mexico, the therapeutic scheme has been weight-adjusted. A DXM dose of $0.12 \mathrm{mg} / \mathrm{kg}$ is administered for 3 days, followed by a dose of $0.06 \mathrm{mg} / \mathrm{kg}$ for 7 days. If the current approach yields fewer adverse effects and does reach the CNS to control neuroinflammation as we hypothesized, there will be direct interest to extend this protocol to several COVID hospitals of the National Health System in Mexico. In addition, increasing the initial sample size (preliminary results) will be required to publish the study and share it with the International scientific community.

\section{Abbreviations}

COVID-19: Coronavirus disease 19; CNS: Central nervous system; GCs: Glucocorticoids; NEWS-2: National Early Warning Score-2; COFEPRIS: Comisión Federal para la Protección contra Riesgos Sanitarios; SARS-CoV-2: Severe acute respiratory syndrome coronavirus 2; RNA: Ribonucleic acid; GFAP: Glial fibrillary acidic protein; NfL: Neurofilament light protein; PAMPs: Pathogen-associated molecular patterns;

DAMPs: Damage-associated molecular patterns; ACE2: Angiotensin-

Converting Enzyme 2; TMPRSS2: Transmembrane Serine Protease 2; NRP-

1: Neuropilin -1; TLR: Toll-like receptor; IL-1 $\beta$ : Interleukin-1 $\beta$; TNFa: Tumor Necrosis Factor a; ROS: Reactive oxygen species; P2X7: Purinergic Receptor P2X7; DVC: Dorsal vagal complex; NTS: Nucleus Tractus solitary; HPA: Hypothalamic-pituitary-adrenal; RVM: Rostral ventrolateral medulla; ACTH: Adrenocorticotrophic hormone; NF-KB: Nuclear Factor Kappa-LightChain-Enhancer of activated B cells; AP-1: Activating Protein-1;

ATP: Adenosine Triphosphate; NLRP3: NLR Family Pyrin Domain Containing 3; DXM: Dexamethasone; BBB: Blood-brain barrier; RBD: Receptor-binding domain; NETs: Neutrophils extracelullar traps; ARDS: Acute respiratory distress syndrome; CO-RADS: Coronavirus-19 Reporting and Data System; RTPCR: Reverse transcription polymerase chain reaction; AIDS: Acquired immunodecficiency syndrome; CRF: Case report format; HGMEL: Hospital General de México Dr. Eduardo Liceaga

\section{Acknowledgements}

We thank the LABNALCIT-UNAM (CONACYT) for the technical support in the acquisition (and/or sorting) of flow cytometry samples. We are grateful to the Unidad de Investigacion at Facultad de Medicina Veterinaria y Zootecnia, UNAM, for the use of its facilities for sample processing. We also thank to MD Pedro Garduño Manjarrez and to Rector de La Universidad Del Ejército y Fuerza Aérea General David Rivera Medina for promoting the participation of SEDENA in this protocol study.

\section{Study status}

The study was registered under the platform Clinical Trials from NCBI in August 2020. It was approved by COFEPRIS in its version \#7 on June 11, 2021 (identifier DI/20/407/04/36). COFEPRIS is a Mexican federal commission for the protection against sanitary risks. Participants are currently being recruited. 


\section{Authors' contributions}

All authors read and approved the final manuscript. Study concept and design: GC, ES, HB, MR, JH, MCC. Data acquisition, allocation, and interpretation: GC, ES, HB, MR, JH, MCC, AJR, DAM, MFMM, LVTA, RLBC, RMW, LERG, KIC, EGV, MRC, YL, MLHM, MLH, KMQ, ASM, SHD, IGRZM, AMC, INMS, EBS, AFP, MJFM, PSHH, JC, LH, NAF, MH, MPT, GM, HJ, EEA, GR, ROA, SOF, SRM, JAHA, JCT, AFR, HB, MCR, RJB, GS, JLA, GF, JPL.RIAR, DMR, LRRA, RAAB, AFR, EAS. Manuscript drafting: GC, ES, HB, MR, JH, JAHA, MCR, RJB, GS, JLA, GF, JPL. Critical revision of the manuscript for important intellectual content: GC, ES, HB, MR, JH, JAHA, MCR, RJB, GS, JLA, GF, JPL. The information obtained from this protocol will be used only for scientific purpose.

\section{Funding}

This work was supported by Dirección General de Personal Académico, UNAM (DGAPA-UNAM, PAPIIT IV201020), by the Institutional program "Programa de Investigación para el Desarrollo y la Optimización de Vacunas, Inmunomoduladores y Métodos Diagnósticos del Instituto de Investigaciones Biomédicas" UNAM as well by the Mexican Ministry of Foreing Affairs (Secretaria de Relaciones Exteriores) and Mexican Agency for International Development Cooperation (AMEXCID) with identifier: 318.01 fund MEX-CHI.

\section{Availability of data and materials}

Data and materials are not available at this moment, because the work being considered is the first approach to a clinical trial currently started. When the study will be completed, the dataset obtained and analyzed will be available from the corresponding author only by reasonable request.

\section{Declarations}

\section{Ethics approval and consent to participate}

This study was reviewed and approved by the Committees of Ethics, Research, and Biosecurity of the five hospital committees: Hospital General de México "Dr. Eduardo Liceaga" (DI/20/407/04/36), Instituto Nacional de Neurología y Neurocirugía (INNN 31/20), Instituto Nacional de Cardiología (INCICh: 20-1167), temporary unit for COVID Citibanamex (FM/DI/107/SR/ 2020), and Hospital Central Militar (055/2021)

All participants will provide written informed consent before enrollment, and all the work will be conducted according to the Helsinki statements.

\section{Consent for publication}

Not applicable

\section{Competing interests}

The authors have no other relevant affiliations or financial involvement with any organization or entity with a financial interest in or financial conflict with the subject matter or materials discussed in the manuscript apart from those disclosed.

\section{Author details}

${ }^{1}$ Instituto Nacional de Neurología y Neurocirugía (INNN), Av. Insurgentes Sur 3877, La Fama, Tlalpan, 14269 Mexico, Mexico. ²Unidad de Investigación UNAM-INC, Instituto Nacional de Cardiología Ignacio Chávez and Instituto de Investigaciones Biomédicas, Universidad Nacional Autónoma de México, Juan Badiano No. 1, Col. Sección XVI, Tlalpan, 14080 Mexico, Mexico. ${ }^{3}$ Clinical Pharmacology Unit, Hospital General de México Dr. Eduardo Liceaga, Dr. Balmis 148, Doctores, Cuauhtémoc, 06720 Mexico, Mexico. ${ }^{4}$ Instituto Nacional de Cardiología Ignacio Chávez, Juan Badiano 1, Belisario Domínguez Secc 16, Tlalpan, 14080 Mexico City, CDMX, Mexico. ${ }^{5}$ Facultad de Medicina, Universidad Nacional Autónoma de México, 04510 Mexico, Mexico. ${ }^{6}$ Unidad Temporal COVID-19, Centro Citibanamex, Avenida del Conscripto 311, Lomas de Sotelo, 11200 Miguel Hidalgo, CDMX, Mexico. ${ }^{7}$ Hospital Militar, Secretaría de la Defensa Nacional, Periférico Blvrd Manuel Ávila Camacho s/n, Militar, Miguel Hidalgo, 11200 Mexico City, CDMX, Mexico. ${ }^{8}$ Departamento de Inmunología, Instituto de Investigaciones Biomédicas, Universidad Nacional Autónoma de México, Circuito escolar s/n, 04510 Mexico, Mexico. ${ }^{9}$ Unidad de Desarrollo e Investigación en Bioprocesos, Escuela Nacional de Ciencias Biológicas del Instituto Politécnico Nacional, Prolongación de Carpio y Plan de Ayala S/N, Col. Casco de Santo Tomas, Del. Miguel Hidalgo, 11340 Mexico, Mexico. ${ }^{10}$ Instituto de Diagnóstico y Referencia Epidemiológicos Dr. Manuel Martínez Báez, Francisco de Miranda 177, Lomas de Plateros, Álvaro
Obregón, 01480 Mexico, Mexico. ${ }^{11}$ Facultad de Medicina, Universidad Autónoma del Estado de Morelos, Avenida Universidad No. 1001, Chamilpa, 62209 Cuernavaca, Morelos, Mexico. ${ }^{12}$ Universidad Autónoma de San Luis Potosí, Álvaro Obregón 64, Col. Centro, C.P. 78000, San Luis Potosí, S.L.P, Mexico. ${ }^{13}$ Laboratorio Nacional de Citometría de Flujo, Instituto de Investigaciones Biomédicas, UNAM, 04510 Mexico, Mexico. ${ }^{14}$ Institute of Physiology and Pathophysiology, Emil-Mannkopff-Straße 2 u, 35037 Marburg Germany. ${ }^{15}$ Departamento de Fisiología, Biofísica y Neurociencias, Centro de Investigación y Estudios Avanzados del Instituto Politécnico Nacional, Av. Instituto Politécnico Nacional 2508, San Pedro Zacatenco, Gustavo A. Madero, 07360 Mexico, Mexico. ${ }^{16}$ Facultad de Química, Universidad Nacional Autónoma de México, 04510 Mexico, Mexico. ${ }^{17}$ School of Medicine, University of Texas Rio Grande Valley - UTRGV, 1201 W University Dr, Edinburg, TX 78539, USA.

Received: 10 July 2021 Accepted: 1 February 2022

Published online: 14 February 2022

\section{References}

1. Medzhitov R. Origin and physiological role of inflammation. Nature. 2008; 454:428-35. https://doi.org/10.1038/nature07201.

2. Li YC, Bai WZ, Hashikawa T. The neuroinvasive potential of SARS-CoV2 may play a role in the respiratory failure of COVID-19 patients. J Med Virol. 2020; 92(6):552-5. https://doi.org/10.1002/jmv.25728.

3. Zhou Z, Kang H, Li S, Zhao X. Understanding the neurotropic characteristics of SARS-CoV-2: from neurological manifestations of COVID-19 to potential neurotropic mechanisms. J Neurol. 2020;267(8):2179-84. https://doi.org/10.1 007/s00415-020-09929-7.

4. Kwong NK, Chong K, Mehta PR, Shukla G, Mehta AR. COVID-19, SARS and MERS: a neurological perspective. J Clin Neurosci Churchill Livingstone. 2020;77:13-6. https://doi.org/10.1016/j.jocn.2020.04.124

5. Li YC, Bai WZ, Hirano N, Hayashida T, Hashikawa T. Coronavirus infection of rat dorsal root ganglia: ultrastructural characterization of viral replication, transfer, and the early response of satellite cells. Virus Res. 2012;163(2):62835. https://doi.org/10.1016/j.virusres.2011.12.021.

6. Desforges M, Le coupanec A, Dubeau P, Bourgouin A, Lajoie L, Dubé M, et al. Human coronaviruses and other respiratory viruses: underestimated opportunistic pathogens of the central nervous system. Viruses. 2019;12(1):14

7. Gasmi A, Noor S, Menzel A, Dosa A, Pivina L, Bjoorklund G. Obesity and insulin resistance: associations with chronic inflammation, genetic and epigenetic factors. Curr Med Chem. 2020;28(4):800-26. https://doi.org/10.21 74/0929867327666200824112056.

8. Danlin L, Richardson G, Benli FM, Park C, de Souza JV, Bronowska AK, et al. Inflammageing in the cardiovascular system: mechanisms, emerging targets, and novel therapeutic strategies. Clin Sci (Lond). 2020;134(17):2243-62. https://doi.org/10.1042/CS20191213.

9. Meinhardt J, Radke J, Dittmayer C, Franz J, Thomas C, Mothes R, et al. Olfactory transmucosal SARS-CoV-2 invasion as a port of a central nervous system entry in individuals with COVID-19. Nat Neurosci. 2021;24(2):1698175. https://doi.org/10.1038/s41593-020-00758-5.

10. Dos Santos MF, Devalle S, Aran V, Capra D, Roque NR, Coelho-Aguiar JM. Spohr TCLSE, Subilaga JG, Pereira CM, D'Andrea Meira I, Niemeyer Soares Filho P, Moura-Neto V. Neuromechanisms of SARS-CoV-2: a review. Front Neuroanat. 2020;14:37

11. Somolon IH, Normandin E, Bhattacharyya S, Mukerji SS, Kellner K, Ali AS, et al. Neuropathological features of COVID-19. N Engl J Med. 2020;383(10): 989-92. https://doi.org/10.1056/NEJMc2019373.

12. Paniz-Mondolfi A, Bryce C, Grimes Z, Gordon RE, Reidy J, Lednicky J, et al. Central nervous system involvement by severe acute respiratory syndrome coronavirus-2 (SARS-CoV-2). J Med Virol. 2020;92(7):699-702. https://doi. org/10.1002/jmv.25915.

13. Kanberg N, Ashton NJ, Andersson LM, Yilmaz A, Lindh M, Nilsson S, et al. Neurochemical evidence of astrocytic and neuronal injury commonly found in COVID-19. Neurology. 2020;95(12):e1754-9. https://doi.org/10.1212/WNL 0000000000010111.

14. Andrews MG, Mukhtar T, Enze UC, Simoneau CR, Perez $Y$, Mostajo-Radji MA, et al. Tropism of SARS-CoV-2 for developing human cortical astrocytes. Version 1. bioRxiv. Preprint. 2021 Jan;18. https://doi.org/10.1101/2021.01.1 7.427024 .

15. Ramani A, Müller L, Ostermann PN, Gabriel E, Abida-Islam P, MüllerSchiffmann A, et al. SARS-CoV-2 targets neurons of 3D human brain 
organoids. EMBO J. 2020;39(20):e106230. https://doi.org/10.15252/embj.202 0106230.

16. Song E, Ce Z, Israelow B, Lu-Culligan A, Veites Prado A, Skriabine S, et al. Neuroinvasion of SARS-CoV-2 in human and mouse brain. J Exp Med. 2021; 218:e20202135.

17. Siddigi HS, Mehra MR. COVID-19 illness in native and immunosuppressed states: a clinical-therapeutic staging proposal. J Heart Lung Transplant. 2020; 39(5):405-7. https://doi.org/10.1016/j.healun.2020.03.012.

18. Hoffmann M, Kleine-Weber H, Schroeder S, Krüger N, Herrler T, Erichsen S, et al. SARS-CoV-2 Cell Entry Depends on ACE2 and TMPRSS2 and is blocked by a clinically proven protease inhibitor. Cell. 2020;181(2):271-280.e8. https://doi.org/10.1016/j.cell.2020.02.052

19. Sungnak W, Huang N, Becavin C, et al. SARS-CoV-2 entry factors are highly expressed in nasal epithelial cells together with innate immune genes. Nat Med. 2020;26(5):681-7. https://doi.org/10.1038/s41591-020-0868-6.

20. Cantuti-Castelvetri L, Ojha R, Pedro LD, Djannatian M, Franz J, Kuivanen S, et al. Neuropilin-1 facilitates SARS-CoV-2 cell entry and infectivity. Science. 2020;370(6518):856-60. https://doi.org/10.1126/science.abd2985.

21. Davies J, Randeva HS, Chatha K, Hall M, Spandidos DA, Karteris E, et al. Neuropilin-1 as a new potential SARS-CoV-2 infection mediator implicated in the neurologic features and central nervous system involvement of COVID-19. Mol Med Rep. 2020;22(5):4221-6. https://doi.org/10.3892/mmr.202 0.11510 .

22. Butowt R, Bilinska K. SARS-CoV-2: Olfaction, brain infection and the urgent need for clinical samples allowing earlier virus detection. ACS Chem Neurosci. 2020;11(9):1200-3. https://doi.org/10.1021/a cschemneuro.0c00172.

23. Yap JKY, Moriyama M, Iwasaki A. Inflammasomes and pyroptosis as therapeutic targets for COVID-19. J Immunol. 2020;205(2):307-12. https://doi. org/10.4049/jimmunol.2000513.

24. Freeman TL, Swartz TH. Targeting the NLRP3 inflammasome in severe COVID-19. Front Immunol. 2020;11:1518. https://doi.org/10.3389/fimmu.2020. 01518.

25. Ribeiro Dem Oliveira-Giacomelli A, Glaser T, Arnaud-Sampaio VF, Andrejew R, Diekmann L, Baranova J, et al. Hyperactivation of P2X7 receptors as a culprit of COVID-19 neuropathology. Mol Psychiatry. 2020;26(4):1-16. https:// doi.org/10.1038/s41380-020-00965-3.

26. Netland J, Meyerholz DK, Moore S, Cassell M, Perlman S. Severe acute respiratory syndrome coronavirus infection causes neuronal death in the absence of encephalitis in mice transgenic for human ACE2. J Virol. 2008; 82(15):7264-75. https://doi.org/10.1128/JVI.00737-08.

27. Wu Y, Xu X, Chen Z, Duan J, Hashimoto K, Yang L. Nervous system involvement after infection with COVID-19 and other coronaviruses. Brain Behav. Immun. 2020;20:30357-3. https://doi.org/10.1016/j.bbi.2020. 03.031 .

28. Zhou L, Zhang M, Wang J, Gao J. Sars-Cov-2: underestimated damage to nervous system. Travel Med Infect Dis. 2020;36:101642. https://doi.org/10.1 016/j.tmaid.2020.101642.

29. Sun T, Guan J. Novel coronavirus and central nervous system. Eur. J. Neurol. 2020;27(9). https://doi.org/10.1111/ene.14227.

30. Cai Y, Hay M, Bishop VS. Synaptic connections and interactions between area postrema and nucleus tractus solitarius. Brain Res. 1996;724(1):121-4. https://doi.org/10.1016/0006-8993(96)00282-X.

31. Goodchild AK, Moon EA. Maps of cardiovascular and respiratory regions of rat ventral medulla: focus on the caudal medulla. J Chem Neuroanat. 2009; 38(3):209-21. https://doi.org/10.1016/j.jchemneu.2009.06.002.

32. Delgado-Roche L, Mesta F. Oxidative stress as key player in severe acute respiratory syndrome coronavirus (SARS-CoV) infection. Arch Med Res. 2020; 51(5):384-7. https://doi.org/10.1016/j.arcmed.2020.04.019.

33. Hamrahian AH. Fleseriu M; AACE adrenal scientific committee. Evaluation and management of adrenal insufficiency in critically ill patients: disease state review. Endocr Pract. 2017;23(6):716-25. https://doi.org/10.4158/EP16 720.RA.

34. Mateos Moreno L, Palacios Garcia N, Estrada Garcia FJ. Adrenal insufficiency in critical patients: new ethiopathogenic concepts and therapeutic implications. Endocrinol Diabetes Nutr. 2017;64(10):557-63. https://doi.org/1 0.1016/j.endinu.2017.09.004

35. Hamming I, Timens W, Bultuis MLC, Lely AT, Navis GJ, van Goor H. Tissue distribution of ACE2 protein, the functional receptor for SARS coronavirus. A first step in understanding SARS pathogenesis. J Pathol. 2004;203(2):631-7. https://doi.org/10.1002/path.1570.
36. Ding YQ, Wang HJ, Shen H, Li ZG, Geng J, Han HX, et al. Study on etiology and pathology of severe acute respiratory syndrome. Zhonghua Bing Li Xue Za Zhi. 2003;32(3):195-200.

37. Leow MKS, Kwek DSK, Ng AWK, Kaw GJL, Lee LSU. Hypocortisolism in survivors of severe acute respiratory syndrome (SARS). Clin Endocrinol (Oxf). 2005:63:197-202.

38. Alzahrani AS, Mukhtar N, Aljomaiah A, Aljamei H, Barkhsh A, Alsudani N, et al. The Impact of COVID-19 Viral Infection on the Hypothalamic-PituitaryAdrenal Axis. Endocr Pract. 2021;27(2):83-9. https://doi.org/10.1016/j.eprac.2 020.10.014.

39. Pal R. COVID-19, hypothalamo-pituitary-adrenal axis and clinical implications Endocrine. 2020;68(2):251-2. https://doi.org/10.1007/s12020-020-02325-1.

40. Coutinho AE, Chapman KE. The anti-inflammatory and immunosuppressive effects of glucocorticoids, recent developments and mechanistic insights. Mol Cell Endocrinol. 2011;335(1):2-13. https://doi.org/10.1016/j.mce.2010.04.005.

41. Van Der Velden VHJ. Glucocorticoids: mechanisms of action and antiinflammatory potential in asthma. Mediators inflamm. 1998;(7):229-37.

42. Ronchetti S, Migliorati G, Bruscoli S, Riccardi C. Defining the role of glucocorticoids in inflammation. Clin Sci (Lond). 2018;132(14):1529-43. https://doi.org/10.1042/CS20171505 PMID: 30065045.

43. Pinto A, Jacobsen M, Geoghegan PA, Cangelosi A, Cejudo ML, Tironi-Farinati $C$, et al. Dexamethasone rescues neurovascular unit integrity from cell damage caused by systemic administration of shiga toxin 2 and lipopolysaccharide in mice motor cortex. PLoS ONE. 2013;8(7):e70020. https://doi.org/10.1371/journal.pone.0070020.

44. RECOVERY Collaborative Group, Horby P, Lim WS, Emberson JR, Mafham M, Bell JL, et al. Dexamethasone in hospitalized patients with Covid-19Preliminary report. N Engl J Med. 2021;384(8):693-704.

45. Erdó F, Bors LA, Farkas D, Bajza A, Gizurarson S. Evaluation of intranasal delivery route of drug administration for brain targeting. Brain Res Bull. 2018;143:155-70. https://doi.org/10.1016/j.brainresbull.2018.10.009.

46. Meneses G, Cárdenas G, Espinosa A, Rassy D, Pérez-Osorio IN, Bárcena B, et al. Sepsis: developing new alternatives to reduce neuroinflammation and attenuate brain injury. Ann N Y Acad Sci. 2019;1437(1):43-56. https://doi. org/10.1111/nyas. 13985 .

47. Espinosa A, Meneses G, Chavarría A, Mancilla R, Pedraza-Chaverri J, Fleury A, et al. Intranasal dexamethasone reduces mortality and brain damage in a mouse experimental ischemic stroke model. Neurotherapeutics. 2020;17(4): 1907-18. https://doi.org/10.1007/s13311-020-00884-9.

48. Meneses G, Gevorkian G, Florentino A, Bautista MA, Espinosa A, Acero G, et al. Intranasal delivery of dexamethasone efficiently controls LPS-induced murine neuroinflammation. Clin Exp Immunol. 2017;190(3):304-14. https:// doi.org/10.1111/cei.13018.

49. Rassy D, Bárcena B, Pérez-Osorio IN, Espinosa A, Peón AN, Terrazas LI, et al. Intranasal methylprednisolone effectively reduces neuroinflammation in mice with experimental autoimmune encephalitis. J Neuropathol Exp Neurol. 2020;79(2):226-37. https://doi.org/10.1093/jnen/nlz128.

50. Zhang Y, Hu S, Wang J, Xue Z, Wang C, Wang N. Dexamethasone inhibits SARS-CoV-2 spike pseudotyped virus viropexis by binding to ACE2. Virology. 2021;554:83-8. https://doi.org/10.1016/j.virol.2020.12.001.

51. Finney $\sqcup$, Glaville N, Farne $H$, et al. Inhaled corticosteroids downregulate the SARS-CoV-2 receptor ACE2 in COPD through suppression of type I interferon. BioRxiv. 2020. https://doi.org/10.1101/202.06.13.149039.

52. Wang $T$, Zhao Y, Fan F, Hu R, Jin X. Dexamethasone inhibits S. aureusinduced neutrophils extracellular pathogen-killing mechanism, possibly through toll-like receptor regulation. Front Immunol. 2017;8:60. https://doi. org/10.3389/fimmu.2017.00060.

53. Zuo Y, Yalavarthi S, Shi H, Gockman K, Zuo M, Madison JA, et al. Neutrophils extracellular traps (NETs) as markers of disease severity in COVID-19. medRxiv. 2020;2020(04):09.20059626.

54. Middleton EA, He XY, Denorme F, Campbell RA, Ng D, Salvatore SP, et al. Neutrophils extracellular traps (NETs) contribute to immunothrombosis in COVID-19 acute respiratory distress syndrome. Bloods. 2020;136(10):1169-79 blood.2020007008.

55. Zhang K, Zhang X, Ding W, Xuan N, Tian B, Huang T, et al. The prognostic accuracy of National Early Warning Score 2 on predicting clinical deterioration in patients with COVID-19: a systematic review and metaanalysis. Front Med (Lausanne). 2021;8:699880. https://doi.org/10.3389/ fmed.2021.699880

56. Baker KF, Hanrath AT, van der Loeff IS, Kay LJ, Back J, Duncan CJ. National Early Warning Score 2 (NEWS2) to identify inpatient COVID-19 deterioration: 
a retrospective analysis. Clin Med (Lond). 2021;21(2):84-9. https://doi.org/10. 7861/clinmed.2020-0688.

57. Harris PA, Taylor R, Thielke R, Payne J, Gonzalez N, Conde JG. Research electronic data capture (REDCap) - a metadata-driven methodology and workflow process for providing translational research informatics support. J Biomed Inform. 2009;42(2):377-81. https://doi.org/10.1016/j.jbi.2008.08.010.

58. Harris PA, Taylor R, Minor BL, Elliott V, Fernandez M, O'Neal L, et al. REDCap Consortium, The REDCap consortium: building an international community of software partners. J Biomed Inform. 2019. https://doi.org/10.1016/j.jbi.201 9.103208 .

59. Thompson $W$. The problem of negative estimates of variance components. Ann Math Stat. 1963;33(1):273-89. https:/doi.org/10.1214/aoms/1177704731.

60. Kenward MG, Roger J. Small sample inference for fixed effects from restricted maximum likelihood. Biometrics. 1997;53(3):983-97. https://doi. org/10.2307/2533558.

\section{Publisher's Note}

Springer Nature remains neutral with regard to jurisdictional claims in published maps and institutional affiliations.

Ready to submit your research? Choose BMC and benefit from:

- fast, convenient online submission

- thorough peer review by experienced researchers in your field

- rapid publication on acceptance

- support for research data, including large and complex data types

- gold Open Access which fosters wider collaboration and increased citations

- maximum visibility for your research: over $100 \mathrm{M}$ website views per year

At BMC, research is always in progress.

Learn more biomedcentral.com/submissions 